\title{
Early Detection of Thyroid Gland Disorders for Students in the Faculty of Applied Medical Sciences
}

\author{
Saeed Bafaraj, Ibrahim Abbas, Saddig Jastaniah, Hanan Abbas, Alamin Musa \\ Department of Diagnostic Radiology, Faculty of Applied Medical Sciences, King Abdulaziz University, \\ Jeddah, Saudi Arabia \\ Email: smbafaraj@kau.edu.sa
}

Received 3 March 2015; accepted 18 March 2015; published 19 March 2015

Copyright (C) 2015 by authors and Scientific Research Publishing Inc.

This work is licensed under the Creative Commons Attribution International License (CC BY). http://creativecommons.org/licenses/by/4.0/

c) (i) Open Access

\begin{abstract}
In this study 70 male students were participated to determine the thyroid disorder through ultrasonography. Thyroid scan was done in $\mathbf{7 0}$ students prospectively with no indicative of thyroid disease (age of 19 - 23 yrs). Thyroid scan for students who participated the study took place in the department of Faculty of Applied Medical Sciences, King Abdulaziz University by using an IU 22 Philips ultrasound machine with a $5-12 \mathrm{MHz}$ linear transducer. Among the total number of the subjects, $26 \%$ was found with abnormal ultrasound findings, $17 \%$ of them with cystic nodule, while solid and mixed nodule represented $4 \%$ for each. The high rate of abnormal findings of thyroid gland in the study suggested that screening using ultrasound scan was useful in detecting early thyroid disorders.
\end{abstract}

\section{Keywords}

Thyroid Disorders, Thyroid Sonography, Thyroid Nodule

\section{Introduction}

\subsection{Background}

The thyroid gland is the first one of the body's endocrine glands, which is developed on approximately the 24th day of gestation. The cells in the thyroid gland have two distinct embryological origins. The major part developed from epithelial cells originating in the endodermal of the primitive pharynx [1]. The thyroid initially develops from caudal to the tuberculumimpar, which is also known as the median tongue bud. This embryonic swelling arises from the first pharyngeal arch and occurs at midline on the floor of the developing pharynx [1]. 
The sonographic's examination of thyroid gland are considered much safer and easier for application and patient preparation, and early detection of disorders of the thyroid gland in the target age group will lead to early treatment of discovered cases, which will improve life status, academic progress and make students get success.

\subsection{Objectives}

To find out: What kind of thyroid disorder could the screening study detect?; How serious the detected condition is?; How much this screening study assist the subject group in receiving early detection and treatment.

\subsection{Materials and Methods}

A prospective study was carried out on 70 male undergraduate students aged 19 - 23 years old. The sonographic scans of those subjects were done in ultrasound division at department of Faculty of Applied Medical Sciences, King Abdulaziz University as a screening based study. Ethical approval was obtained from the medical research of the university. The examination was explained to the students and consent was obtained.

Demographic subject's data including their age and gender, and the ultrasound findings of thyroid gland were collected and recorded on a data collecting sheet before subject leaving the ultrasound department.

Including criteria: male students of Faculty of Applied Medical Sciences (FAMS) are included in the screening study.

Excluding criteria: only those who have been clinically diagnosed with thyroid disorder prior the study are excluded.

\section{Literature}

\subsection{The Relation between Thyroid Nodules and Thyroid Malignancy Incidences}

A palpated mass may be solid, cystic, or mixed in nature. Benign cysts can be evacuated successfully by aspiration. The aspirated fluid is usually clear yellow or bloody with high levels of thyroid hormones. A true cyst has a very low risk of malignancy. However, the presence of a cyst does not exclude neoplasia, especially if the mass is mixed. Desjardins et al. found that one half of their patients with thyroid carcinoma had a cystic component in the tumor [2].

Nodules can be visualized as areas of increased (hot) or decreased (cold) tracer activity. A nonfunctioning (cold) nodule placed in the center of a lobe with functioning normal tissue superficial to it may appear as warm on scans because of integrated activity with depth. Unless oblique views are imaged, the presence of activity concentration cannot be definitively determined. Therefore, nodules should be identified as being functioning (hot), nonfunctioning (cold), or photon deficient. Hot nodules which are typically adenomas are more often benign than cold lesions [3] [4].

Ultrasound is a valuable diagnostic tool in assessing the following indications:

- Classification of a palpated lump. eg solid, cystic, mixed;

- Evaluate adjacent structures;

- Determining the location of a palpable lump (within or outside of the thyroid;

- Identifying a cause for Hyperthyroidism;

- Post surgical complications (e.g., abscess, oedema);

- Multi Nodular Goiter (MNG): Follow up nodules;

- Guidance of injection, aspiration or biopsy;

- Relationship of normal anatomy and pathology to each other [5].

\subsection{Preparation}

- Low collared shirt;

- Remove jewellery around the neck;

- Towel across the shoulders/chest;

- Lie the patient so their head is at the top of pillow and tipped right back;

- A pillow or towel can be placed under the shoulders [5]. 


\subsection{Equipment Selection}

- A 7 - $14 \mathrm{MHz}$ linear transducer;

- Deep seated tumours, retrosternal thyroids or large patients may require a curvi-linear array transducer of 3.5 $\mathrm{MHz}$;

- Good color/power/Doppler capabilities when assessing vessels or vascularity of a structure [5].

\subsection{Ultrasound Scanning Technique}

- Begin with a survey scan in transverse down the midline to assess for tracheal deviation and obvious pathology;

- Tilt the patients head slightly to the contralateral side and scan down in transverse;

- Rotate into longitudinal and scan from medial to lateral;

- Repeat this for the other side with the head tilted the other way;

- With the patients head/neck straight, scan the isthmus in longitudinal and transverse;

- Scan down each side of the neck in transverse for alternative pathology [5].

\subsection{Ultrasound Characteristics Which Suggest a Benign Nodule Include}

- Nodule filled with fluid (likely a cyst);

- Multiple nodules throughout the gland (likely a multinodular goiter);

- No blood flowing through nodule (again, likely a cyst) [6].

\section{Data Analysis and Results}

\subsection{The Frequency of Gender That Participates in the Study}

Figure 1 shows that 70 male participate in the study with $100 \%$.

\subsection{The Frequency of Abnormal Finding}

Figure 2 shows that the abnormal finding of the subject group using ultrasound screening is $26 \%$, while $74 \%$ of the cases are normal.

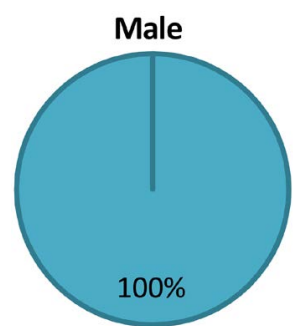

Figure 1. The precentage of gender participates in the study.

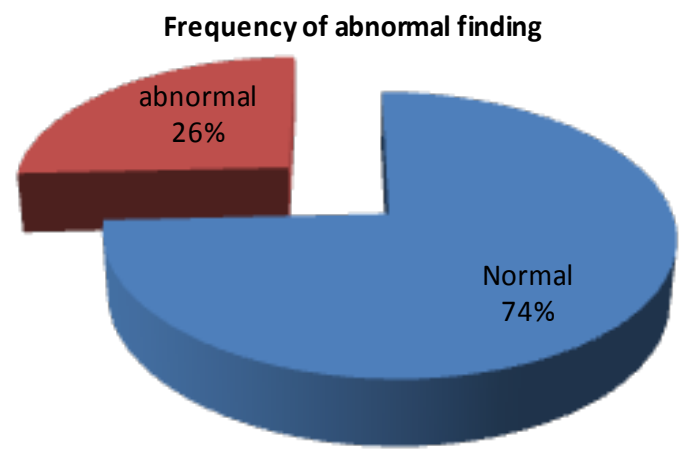

Figure 2. The frequency of abnormal finding. 


\subsection{Sonography Finding for Subject Group}

Figure 3 shows that 52 cases out of 70 are clear of any types of nodule, while 11 cases out of 70 were diagnosed with solitary nodule, and 7 cases out of 70 with multiple nodule

\subsection{Characteristics of Nodules Finding for Subject Group}

Figure 4(a) shows that $75 \%$ of overall cases are clear of any types of nodule, while cystic nodule represents 17\%, and 4\% for both solid and mixed nodule; and Figure 4(b) shows the frequency.

\subsection{Frequency of Thyroiditis \& Parathyroiditis Finding for Subject Group}

In Figure 5(a), 4 cases out of 70 are diagnosed with thyroiditis, and only 2 cases diagnosed with parathyroiditis, and Figure 5(b) shows the percentage.

\subsection{Sonography Positive Finding According to Thyroid Lobes}

Figure 6(a) and Figure 6(b) show that 9 cases (50\%) out of 18 were diagnosed with positive finding abnormalities that affected both right \& left lobes of the thyroid, while 4 out of $18(22 \%)$ cases were diagnosed with positive finding abnormalities of left lobe of the thyroid, and only 5 cases (28\%) was diagnosed with positive finding abnormalities of right lobe of the thyroid.

\section{Discussion}

Carroll, B.A. reported that the prevalence rate of thyroid gland nodules sonographically detected was $13 \%$ [7].

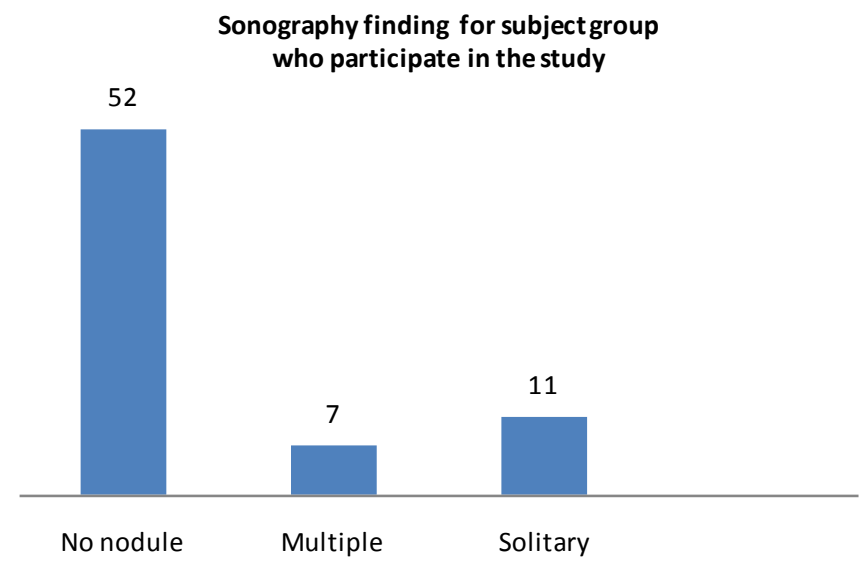

Figure 3. Sonography finding types for subject group.

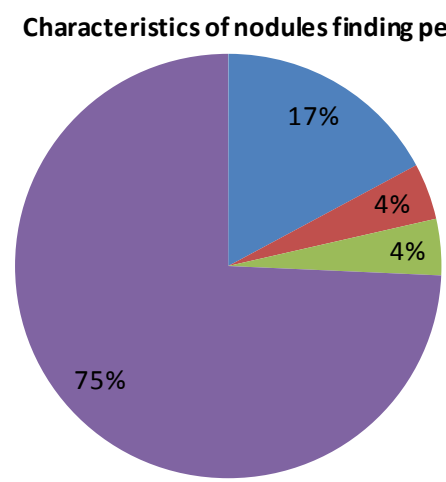

(a)

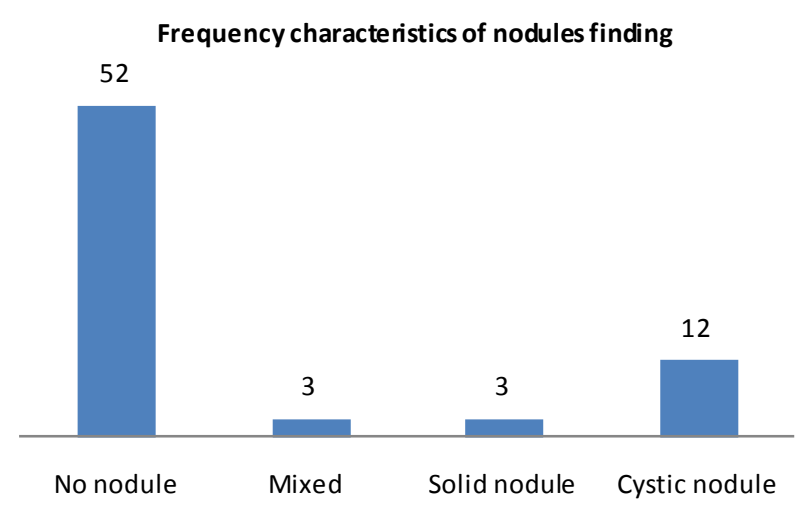

(b)

Figure 4. (a) Characteristics of nodules finding percentage; (b) Characteristics of nodules finding frequency. 


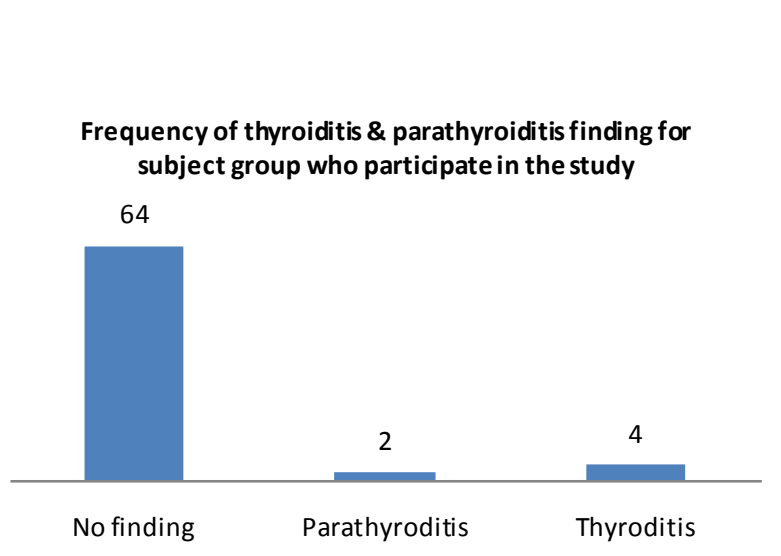

(a)

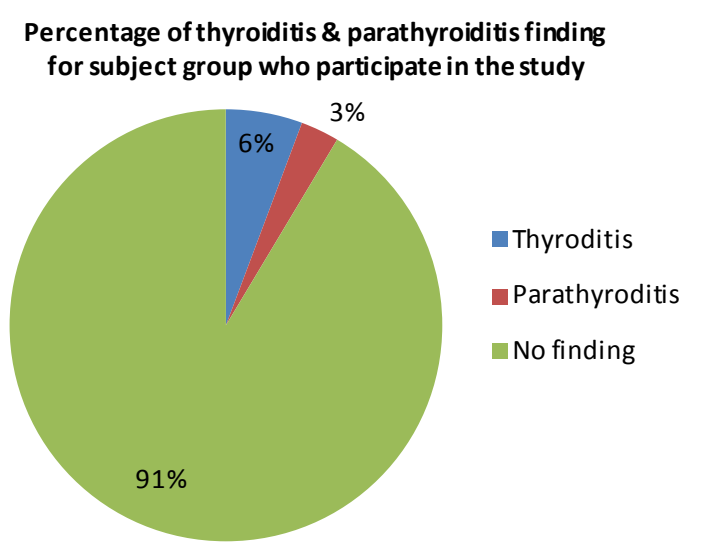

(b)

Figure 5. (a) Frequency of thyroiditis \& parathyroiditis finding; (b) Percentage of thyroiditis \& parathyroiditis finding.

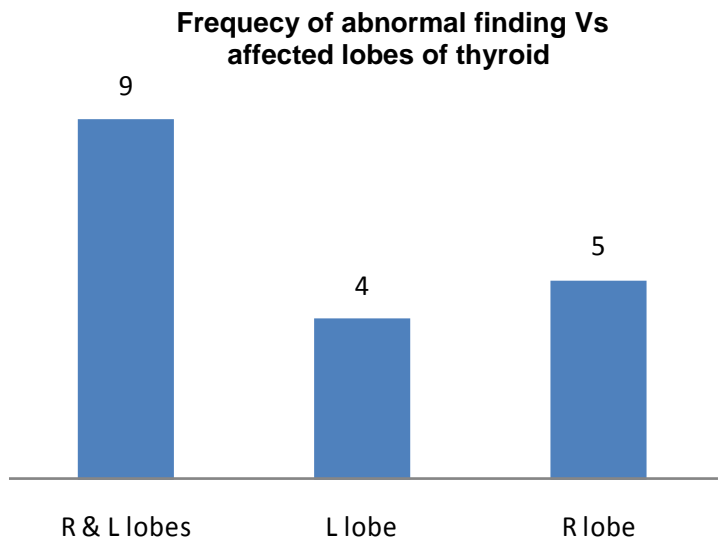

(a)
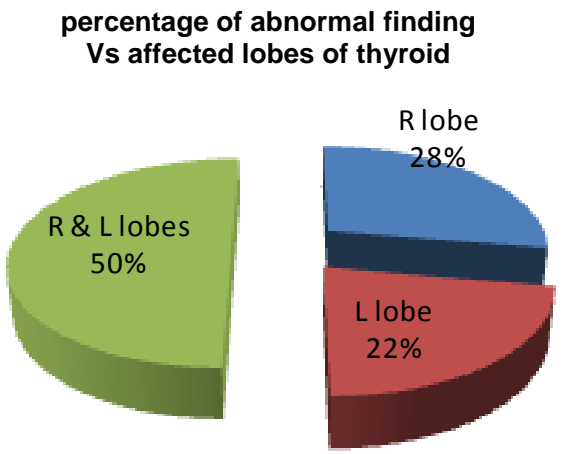

(b)

Figure 6. (a) Frequency of abnormal finding Vs affected lobes of thyroid; (b) Percentage of abnormal finding Vs affected lobes of thyroid.

In the study of Steele et al., incidental thyroid abnormalities were seen in $9.4 \%$ of patients [8].

According to studies of Carrol, B.A. and Steele et al. that has been reported above, the prevalence rate of thyroid nodules by ultrasonography in our subjects was (17\%) significantly greater than the rate they found, and thyroid abnormalities were seen in $26 \%$ of the subject group.

\section{Recommendation}

Ultrasonography can be used as a successful tool for early detection of thyroid disorders.

\section{Acknowledgements}

This project was funded by the Deanship of Scientific Research (DSR), King Abdulaziz University, Jeddah, under grant No. 284/142/1434. The authors, therefore, acknowledge DSR for the technical and financial support.

\section{References}

[1] Zapanta, P.E. and Shokri, T. (2010) Embryology of the Thyroid and Parathyroids, Thyroid Embryology Clinical Correlations. http://emedicine.medscape.com/article/845125-overview\#aw2aab6b5

[2] Can, A.S. and Peker, K. (2008) Comparison of Palpation-Versus Ultrasound-Guided Fine-Needle Aspiration Biopsies in the Evaluation of Thyroid Nodules. BioMed Central Research Notes, 1, 12.

[3] Bruneton, J.N., Balu-Maestro, C., Marcy, P.Y., Melia, P. and Mourou, M.Y. (1994) Very High Frequency (13 MHz) 
Ultrasonographic Examination of the Normal Neck: Detection of Normal Lymph Nodes and Thyroid Nodules. Journal of Ultrasound in Medicine, 13, 87-90.

[4] Nabriski, D., Ness-Abramof, R., Brosh, T., Konen, O., Shapiro, M.S. and Shenkman, L. (2003) Clinical Relevance of Non-Palpable Thyroid Nodules as Assessed by Ultrasound-Guided Fine Needle Aspiration Biopsy. Journal of Endocrinological Investigation, 26, 61-64. http://dx.doi.org/10.1007/BF03345124

[5] http://www.ultrasoundpaedia.com/normal-thyroid.

[6] http://fitsweb.uchc.edu/student/selectives/Luzietti/Thyroid_hyperthyroid.htm

[7] Carroll, B.A. (1982) Asymptomatic thyroid Nodules: Incidental Sonographic Detection. American Journal of Roentgenology, 138, 499-501.

[8] Steele, S.R., Martin, M.J., Mullenix, P.S., Azarow, K.S. and Andersen, C.A. (2005) The Significance of Incidental Thyroid Abnormalities Identified during Carotid Duplex Ultrasonography. Archives of Surgery, 140, 981-985. http://dx.doi.org/10.1001/archsurg.140.10.981 